\title{
TOPIC: ABDOMINAL WALL HERNIA - Epigastric hernia: choice of approach, repair, results, follow up
}

\section{VS:04 \\ LAPAROSCOPIC REPAIR OF MORGAGNI \\ HERNIA: AN INTERESTING CASE WITH HEMOPERICARDIUM AS POSTOPERATIVE COMPLICATION \\ C Maldonado ${ }^{1}$, D Daroch ${ }^{2}$, R Funke ${ }^{3}$, N Jarufe ${ }^{4}$, M Gabrielli ${ }^{5}$ \\ 'Pontificia Universidad Católica de Chile, Santiago, CHILE}

Introduction: Morgagni hernia, the less common of congenital diaphragmatic hernias, corresponding to $3-5 \%$ of the total, is usually diagnosed in childhood, but $5 \%$ of them are diagnosed in adulthood. The aim of this video is to describe the technique of laparoscopic repair of Morgagni hernia in an adult patient who presented a hemopericardium as a postoperative complication.

Methods: A 81-year-old woman presented to the emergency department with a 1-day history of acute epigastric pain, She denied vomiting or other sintoms. She refers this pain for the last 3 months. The pain was treated as functional colopathy with no clear improvement.

Examination showed mild epigastric tenderness. Computed tomography (CT) scan demonstrated an anterior diaphragmatic hernia of Morgagni no complicated. The patient proceeded to a laparoscopic repair of the hernia.

Laparosocopic exploration confirmed the presence of a Morgagni hernia, with a hernia of $5 \mathrm{~cm}$ with fat content. The sac was reduced. The edge of the hernial defect was repaired with a $10 \times 5 \mathrm{~cm}$ polypropylene encapsulated with polydioxanone mesh. This was secured with cardinal points of polypropylene and with $5 \mathrm{~mm}$ absorbable straps around the mesh.

Result: The patient made a good recovery and was discharged 2 days after surgery. She represented 2-week post-op with epigastric pain, shortness of breath. CT scan revealed a hemopericardium. This was drained percutaneously with a pigtail catheter with good clinical improvement.

She stayed 10 days in hospital before being discharged in good conditions and remains well 6 months postoperatively.

Conclusion: Symptomatic patients with a Morgagni hernia should be offered a laparoscopic repair. It is probably preferable to use a mesh and to resect the hernia sac. However, considering the lack of evidence in Morgagni hernias, the risks of complications in the method of mesh fixation are present and need to be considered by the surgeons.

\section{VS:05}

INCOMPLETE EPIGASTRIC HERNIA

F Gorganchian ${ }^{1}$, V Santa Maria ${ }^{1}$, T Dupuy Cash $^{1}$, I Mori' ${ }^{1}$ S Anania ${ }^{1}$ ${ }^{\prime}$ Sanatorio Finochietto, CABA, ARGENTINA

Introduction: An epigastric hernia is a protrusion of pre-peritoneal fat with or without a peritoneal pouch. When the protrusion is only peritoneal fat it's called false hernia and if there is a peritoneal pouch it's named true epigastric hernia. Epigastric hernias are the second most common type of linea alba abdominals defects in adults. They have an incidence rate of $0,5-10 \%$. They occur from the xiphoid process to the umbilicus, most common in man and frequently asymptomatic. When symptomatic it present with epigastric pain and palpable swelling. There is not a unique theory about the cause of epigastric hernia; the oldest is the theory of the perforating vessel of Moschcowitz in 1917 but it has never been proved. We report a case of a false epigastric hernia without palpable swelling in order to discuss a rare finding in the anatomy.

Case Report: A 35 years old female patient with intermittent epigastric pain for 10 years that in the last month has become continuous and so intense that enables hers daily activities. The pain was not related to food ingestion or physical activity. She also refers multiples consultation for this pain over the time and a diagnosis of acid sensitive syndrome and anxiety disorder. The physical examination noted a stabbing pain in the midline of the abdomen located $7 \mathrm{~cm}$ above the umbilicus. No palpable swelling was observed. She had two upper gastrointestinal endoscopies with normal results and several abdominal wall ultra-sonographies with no positives findings. An abdominal tomography was performed and a bilateral Bochdalek hernia was found. Finally a dynamic ultrasonography was performed and a posterior layer defect of $1 \mathrm{~cm}$ of diameter was found with a small protrusion of peritoneal fat but an intact anterior layer. An exploratory laparoscopy was decided, and a small protrusion of the round ligament of the liver was found without a perforation of the anterior facial layer. The defect was closed with an interrupted suture of polidioxanone. Follow up showed no post-operatory pain and a complete remission of the symptoms.

Discussion: The oldest theory about the phisiopatology of an epicastric hernia was postulated by Moschcowitz in 1917 with the report of two cases. He says that epigastric herniation is linked to the role of vascular lacunae at the linea alba. As they pierce the transversalis fascia a layer of fascial fibers is being pushed upwards and a space is created between the peritoneum and the fascia. When the abdominal tension is increased parts of the pre-peritoneal fat is forced to this space. We haven't found a clear vessel but we believe that this was the origin of the partial hernia because we did find a protrusion of the round ligament that was pushing upwards piercing the transversalis fascia.

Normaly an epigastric hernia with a diameter minor to $2 \mathrm{~cm}$ has to be treated with an open approach and without mesh placement, doe this is open to discussion cause the papers that evidence this statement also includes umbilicus hernia. In our case we chose a laparoscopic approach because there was no evidence of a clear defect on the anterior layer of the rectus fascia. In these rare cases it is preferable this approach because it enables a clear view of the defect. 